\title{
Modalidades de uso y razones de éxito de las redes sociales entre los jóvenes universitarios españoles para su aplicación al Espacio Europeo de Eucación Superior (EEES)
}

\author{
Graciela Padilla Castillo \\ gracielapadilla@ccinf.ucm.es \\ Universidad Complutense de Madrid
}

Recibido: $29 / 07 / 2012$

Aceptado: 22/10/2012

\section{Resumen}

El 97,8\% de los jóvenes españoles que va a comenzar sus estudios universitarios utiliza Internet y prácticamente el mismo porcentaje -el 97,7\%- está presente en una o más redes sociales. Los datos se desprenden de un estudio realizado por la consultora Top Position, especializada en comunicación digital, con el asesoramiento de tres profesores de la Universidad Complutense de Madrid. Se trata de una de las primeras aproximaciones científicas y educativas al uso de las redes sociales en España. Relacionando esos datos con la realidad de la docencia universitaria, proponemos algunos cambios o innovaciones educativas, que puedan ayudar a los docentes universitarios a acercarse mejor al alumnado. Las redes sociales están aquí para quedarse. Como parte importante de la vida cotidiana de los jóvenes españoles, la universidad del EEES también debe acercarse a ellas.

Palabras clave: Redes sociales, Facebook, Tuenti, Universitarios, Perfiles en redes sociales.

\section{Social Networks' Use Patterns and Reasons for Success among College Students for its Application to European Higher Education Area (EHEA)}

\begin{abstract}
The $97.8 \%$ of Spanish youth who will start college use Internet and about the same percentage $-97.7 \%$ is present in one or more social networks. The data comes from a study by the consulting firm Top Position, specializing in digital media, with the advice of three professors from the Complutense University of Madrid. This is one of the first scientific and educational approaches to the use of social networks in Spain. Relating these data with the reality of university teaching, we propose some changes or educational innovations that can help teachers' better approach to college students. Social networks are here to stay. As an important part of everyday life of Spanish youth, the EHEA should also approach them.
\end{abstract}

Keywords: Social networks, Facebook, Tuenti, Social networks' profiles.

\section{Referencia normalizada}

PADILLA CASTILLO, Graciela (2012): "Modalidades de uso y razones de éxito de las redes sociales entre los jóvenes universitarios españoles para su aplicación al Espacio Europeo de Eucación Superior (EEES)". Estudios sobre el mensaje periodístico. Vol. 18, núm. especial noviembre, págs.: 671-679. Madrid, Servicio de Publicaciones de la Universidad Complutense.

Sumario: 1. Introducción. 2. Metodología. 3. Desarrollo. 4. Conclusiones. 5. Referencias bibliográficas.

\section{Introducción:}

E1 97,8\% de los jóvenes españoles que va a comenzar la carrera conoce y utiliza habitualmente Internet, y prácticamente el mismo porcentaje -el 97,7\%- está presente en una o más redes sociales. Los datos se desprenden de un estudio realizado por la empresa consultora Top Position (2011: web), especializada en comunicación digital, con el asesoramiento y apoyo de tres profesores de la Universidad Complutense de 
Madrid: Felicísimo Valbuena, Eva Aladro y Graciela Padilla. Se trata de una de las primeras aproximaciones científicas al uso de las redes sociales en España.

El estudio se realizó en dos fases. En la primera, coordinaron cuatro sesiones de grupos focales, cuyo análisis ayudó a preparar el cuestionario de una encuesta posterior, aplicada en una segunda fase. La encuesta se administró en junio de 2011, a 420 jóvenes que estaban examinándose de las pruebas de selectividad. El margen de error es inferior a $+-4,8 \%$, para un grado de confianza de $0,95 \%$. De los jóvenes presentes en las redes sociales, dos de cada tres $(68,7 \%)$, las consideran algo importante o muy importante en su vida. Más del $90 \%$ de los encuestados tiene cuenta en Tuenti y 3 de cada 4 también en Facebook.

Relacionando estos datos con la realidad de la docencia universitaria, planteamos utilizar los resultados del estudio para proponer algunos cambios o innovaciones educativas, que puedan ayudar a los docentes universitarios a acercarse al alumnado. Las redes sociales no son una moda pasajera y como parte importante de la vida cotidiana de los jóvenes españoles, la universidad española del EEES (Espacio Europeo de Educación Superior) también debe acercarse a ellas.

Antes de introducirnos de lleno en el estudio, haremos un repaso por las últimas publicaciones científicas sobre el asunto. Como indicábamos en las primeras líneas, presentamos aquí una de las primeras aproximaciones científicas al uso de las redes sociales en España, ya que no hay suficientes estudios cuantitativos fiables. Es cierto que algunos teóricos se han acercado al asunto, pero casi siempre desde un punto de vista cualitativo y descriptivo.

Sí existe una investigación muy válida sobre las presentaciones audiovisuales en el sistema de correo electrónico en la Red. Fue realizada por el grupo de investigación consolidado UCM número 94820, Estructuras comunicativas e interacciones en los distintos niveles de la Comunicación Interpersonal. Este proyecto formó parte del Programa de Creación y Consolidación de Grupos de Investigación Banco Santander Central HispanoUniversidad Complutense 2008-2010, y llevó como título La comunicación interpersonal y pública de los textos audiovisuales a través del correo electrónico.

La base de aquella investigación fue el análisis de contenido, formal y de usos y motivaciones, además del estudio de los sistemas de transmisión grupal, de 2.000 presentaciones audiovisuales que, mediante programas como PowerPoint, Windows Media Player o con el acceso a YouTube, fueron enviadas, recibidas y difundidas por usuarios españoles de programas de correo electrónico. El grupo de investigación procedió, en una primera instancia, a fijar el contenido de las 2.000 presentaciones realizando dos tareas: a) una primera, de reparto e identificación de esa cantidad de mensajes, entre los miembros del equipo de la investigación y b) una segunda, de control en el análisis de contenido. Aquí, el equipo comprobó que el análisis realizado por todos los investigadores integrantes de este proyecto era acorde. Como referencia fundamental, que da nombre al proyecto investigador, se utilizó la Teoría de la Pirámide de Necesidades de Abraham Maslow (1943-1988). La Pirámide de Maslow representa uno de los arquetipos básicos de la personalidad humana dinámica.

Los resultados fueron difundidos y publicados en varias revistas científicas, durante 2011 y 2012. Parte del grupo explicaba: "Actualmente, abundan las investigaciones 
sobre las redes sociales, los sistemas de interacción mediados por los interfaces de usuarios, y los nuevos fenómenos de identidad en los medios digitales. Sin embargo, no son numerosas las investigaciones sobre las presentaciones audiovisuales en el sistema de correo electrónico en la Red. Hay algunas investigaciones sobre cómo el uso más popular de Internet es, precisamente, la mensajería y las búsquedas de información" (Padilla et al., 2011: 181-195).

También se referían a las investigaciones de diversos autores que muestran los entornos mediáticos creados en Internet: "Estos reemplazan el marco primario de referencia y crean un mundo parasocial esencial en el que el individuo puede percibir al grupo, asociarse con él e incluso, superar sus propias limitaciones sociales previas" (Padilla et al., 2011: 181-195). Como aventuraba Aladro (2009: 107), la comunicación interpersonal establece sistemas informativos, que actúan sobre la consciencia individual.

Todos estos cambios en los marcos de referencia, los mundos parasociales y las relaciones con los demás de cada individuo, son consecuencia de Internet y de las nuevas formas de comunicación cibernéticas entre individuos. Larrañaga (2008: 3) explicaba que "el mayor número de horas de consumo de Internet, disminuye el consumo de otras actividades, pues el individuo tiende a sustituir unas actividades por otras". Por ello, "un mayor uso de la Red afecta al consumo de los medios tradicionales" (Larrañaga, 2008: 3).

Esto no impide que esa afirmación sobre la Red tenga su parte positiva. Precisamente, Castelló (2010: 91), habla de las cuatro C de la Web 2.0 y 3.0: "Compartir, Comunicar, Conversar y Cooperar". Las formas de comunicación interpersonal han cambiado gracias a las redes sociales y con ellas, las formas de comunicación de masas, el consumo de los medios y la publicidad en todos los soportes. Internet ofrece más posibilidades, una publicidad multicanal y un reparto distinto de la tarta publicitaria (Galán y Del Pino, 2011: 3). Ya no basta con producir y contratar los spots tradicionales que se insertan en televisión, entre corte y corte de un programa, película o serie. Ahora hay que estar en las redes sociales. Allí, los anuncios llegan a menos consumidores generalistas, pero más consumidores potenciales y segmentados. Los anunciantes cuentan con una información mucho más cualificada sobre sus públicos, porque el perfil social de cada seguidor contiene toda la información que necesitan.

Esto cambia la lógica empresarial del consumidor, por un lado; y de las empresas, marcas e instituciones, por otro. Matellanes-Lazo (2012: 22) explica que: "Para lograr el éxito en Internet, no es suficiente tener una Web muy bien diseñada, atractiva y con buenos contenidos de información". Del mismo modo, Gómez Nieto et al. (2012: 2) confirman que "la imagen corporativa de las empresas en Internet se ha consolidado como un factor estratégico clave en el ámbito empresarial”. Así, es comprensible que "la inversión publicitaria en medios interactivos haya vivido, en los últimos años, un crecimiento muy rápido, justificado por el incremento de la penetración en la población de Internet así como por las posibilidades de medición del retorno de la inversión que este medio ofrece" (Castelló, 2010: 89). La investigación protagonista de este escrito, y descrita en las líneas siguientes, está unida a todos estos argumentos. 


\section{Metodología}

En nuestra filosofía de apoyar con datos reales las afirmaciones anteriores y no con conjeturas o meras visitas a los perfiles de personas, empresas y marcas en las redes sociales, rescatamos los resultados de un estudio cuantitativo con un ingente trabajo de campo. Como indicábamos al comienzo, fue realizado por la empresa consultora Top Position (2011: web), especializada en comunicación digital y dirigida por Ricardo Carreras, con el asesoramiento y apoyo de tres profesores de la Universidad Complutense de Madrid: Felicísimo Valbuena, Eva Aladro y Graciela Padilla.

Los citados investigadores realizaron el estudio en dos fases. En la primera, coordinaron cuatro sesiones de grupos focales (focus groups), cuyo análisis ayudó a preparar las preguntas de una encuesta posterior, aplicada en la segunda fase. Esta encuesta se administró en junio de 2011, a 420 jóvenes que estaban examinándose de las pruebas de selectividad. El margen de error es inferior a $+-4,8$ por ciento, para un grado de confianza de 0,95 por ciento. Se trata de uno de los pocos estudios científicos acerca del uso de las redes sociales en España y los resultados son tan enriquecedores como sugerentes.

Sólo hay que ver esos resultados. El 97,8 por ciento de los jóvenes españoles que va a comenzar sus estudios universitarios superiores, conoce y utiliza habitualmente Internet. Prácticamente, el mismo porcentaje, 97,7 por ciento, está presente en una o más redes sociales. De esos jóvenes presentes en las redes sociales, dos de cada tres (68,7 por ciento), las consideran algo importante o muy importante en su vida. Los demás ven las redes sociales de esta manera:

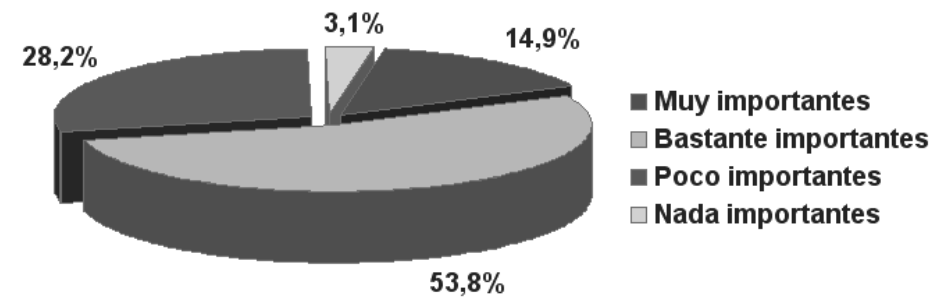

Gráfico 1. Percepción de las redes sociales en las vidas de los jóvenes españoles. Fuente:

Top Position (2011: web).

Más del 90 por ciento de los 420 jóvenes encuestados, tiene cuenta en Tuenti y tres de cada cuatro de ellos también la tiene en Facebook. Respecto a las empresas y marcas presentes en las redes sociales, a los encuestados les parece útil que sus firmas favoritas tengan creados perfiles, y nueve de cada diez consideran que es útil para las empresas, para los usuarios o para ambas partes, de la siguiente forma:

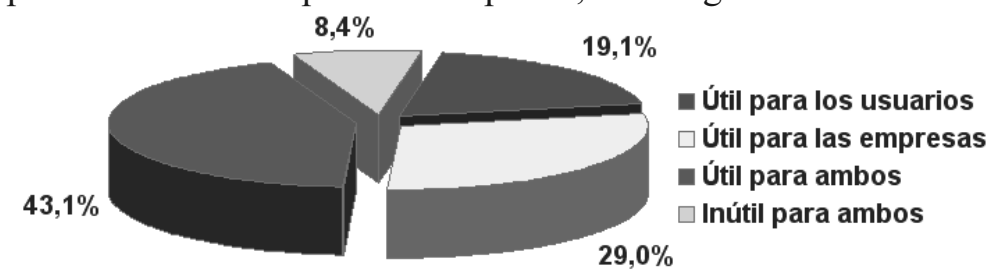

Gráfico 2. Utilidad de las redes sociales para firmas, empresas, instituciones, productos.

Fuente: Top Position (2011: web). 
Preguntados acerca de qué empresa o marca gestiona y cuida mejor sus perfiles, las más mencionadas de forma espontánea son Movistar (24,9 por ciento de las respuestas), Telepizza (casi el 12 por ciento) y Zara (algo menos del 5 por ciento). La televisión aparece con varios ejemplos por debajo de ese 5 por ciento: RTVE, Telecinco, Antena 3, La Sexta, Marca TV, El Hormiguero (entonces de Cuatro) y El internado.

A continuación, el estudio se centra en las dos redes sociales con más impacto en España. Facebook, en primer lugar. Su creador, Mark Zuckerberg, destacó un elemento en su visita a la Universidad de Navarra, en 2008: "el news feed, que definió como una de las piezas clave de su producto gracias a los mecanismos de retroalimentación que genera entre los usuarios" (Torres-Salinas, 2008: 683). Este mecanismo hace que el usuario elija los temas, personas y perfiles que más le interesen para ver su estado y actualizaciones, cada vez que ingrese en el sitio.

Nos interesa citar esta herramienta pensando en todos los perfiles corporativos e institucionales, universidades del EEES incluidas. Sobre todo en la red social con más usuarios en todo el mundo y con más impacto en los jóvenes españoles, según el estudio. Tres de cada cuatro encuestados tienen una cuenta en Facebook. Sus perfiles tienen, de media, 18 meses de antigüedad. Utilizan su cuenta en Facebook varias veces por semana. Además, se conectan una media de 40 minutos por sesión y están conectados, de media, con 100 amigos.

Esta última parte, sobre los contactos, también puede resultar interesante para los perfiles corporativos e institucionales. Las redes sociales permiten una nueva forma de boca-oreja o mouth-marketing, y cuando un usuario se hace seguidor de un perfil, automáticamente todos sus contactos conocen ese hecho y ese perfil. Esto cobra más fuerza según crecen la red social y el número de contactos de cada usuario. Y son muchos contactos, como indica el estudio:

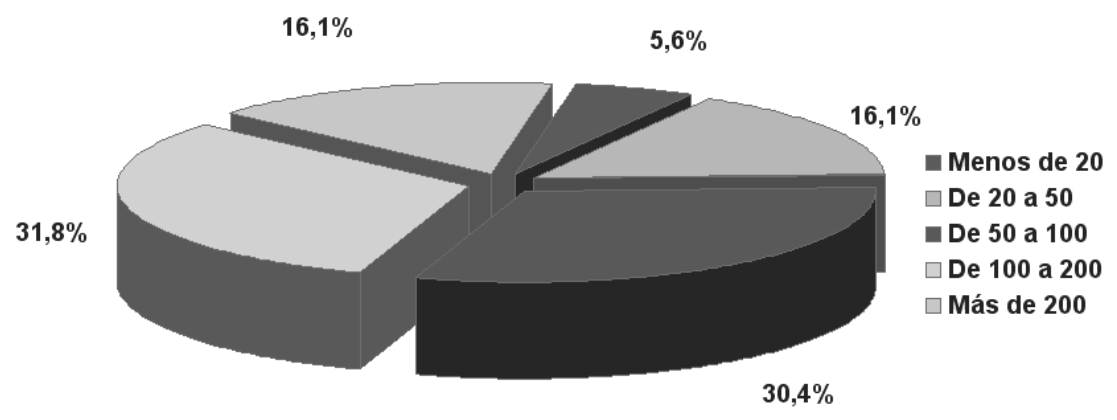

Gráfico 3. Número de amigos en Facebook que tienen los jóvenes encuestados. Fuente: Top Position (2011: web).

Respecto a Tuenti, la red social española creada en 2006 y adquirida por Movistar en agosto de 2010, el estudio revela que los perfiles tienen, de media, 30 meses de antigüedad. La mayoría de los jóvenes encuestados utiliza su cuenta a diario. Además, se conectan una media de 70 minutos por sesión y la mayoría tiene 150 amigos: 


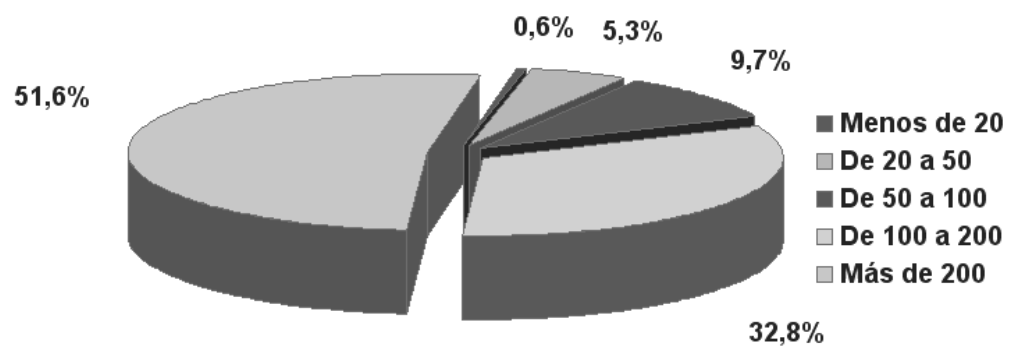

Gráfico 4: Número de amigos en Tuenti que tienen los jóvenes encuestados. Fuente: Top Position (2011: web).

Como decíamos desde el principio, no tiene sentido dar datos de los seguidores de los perfiles en ninguna publicación científica, pues estos datos descriptivos son potencialmente caducos. Sin embargo, los resultados de la encuesta y el estudio, aquí presentados, sí tienen valor porque nos acercan a los intereses reales de los jóvenes españoles. No importan tanto los números, sino el hecho de que queden patentes nuevas filosofías y nuevas formas de relacionarse. La universidad y la docencia universitaria del EEES, como apuntábamos, tienen que aprovechar estos cambios.

\section{Desarrollo}

Los profesores universitarios nos preocupamos por modernizar y transformar la educación continuamente. Claxton (2005: 260) decía que esta reforma debe hacerse por dos razones: una económica y otra personal. Respecto a la razón económica, argumentaba que el conocimiento cambia y los jóvenes deben adaptarse a ese cambio. Para conseguirlo, el docente debe ayudarles a conseguir una mente dúctil y ágil que les permita aprender aquello que sea necesario (Claxton, 2005: 260). Según el autor, sólo así se consiguen personas innovadoras y dotadas de recursos.

Los gobiernos han modificado los horarios de clase, los currícula de los profesores y los métodos de evaluación. Sin embargo, no han conseguido modernizar el corazón de la educación hasta que las Ciencias Sociales empezaron a trabajar sobre el asunto (Claxton, 2005: 260). Todas las pistas estaban en Internet, en la nueva comunicación interpersonal electrónica y por supuesto, en las redes sociales.

El mundo digital afecta a todas las parcelas del trabajo y de las actividades humanas. Las relaciones intersubjetivas han cambiado gracias a la innovación tecnológica. Esta innovación no debe ser contemplada como el agente de cambio en sí mismo, "sino desde los usos sociales y las prácticas de los sujetos que determinan la construcción de sentido alrededor de ella" (Cáceres et al, 2009: 213).

Arras, Torres y García-Valcárcel (2011: 2) hablan de una sociedad marcada por los cambios globales y la innovación tecnológica. Esos dos aspectos influyen en "las estrategias de educación y nuevas estructuras de aprendizaje". Añaden que la sociedad global se construye a través de las TIC (Tecnologías de la Información y la Comunicación) y precisamente, las competencias en TIC son nuevos requisitos de muchos puestos laborales. Por ello, la educación superior de calidad no puede obviarlo y "se plantea el diseño curricular centrado en el aprendizaje y basado en competencias, una de ellas en TIC, 
como una de las alternativas que permiten lograr el objetivo de formación y de pertinencia con relación a los cambios en el entorno" (Arras et al., 2011: 2-3).

Además, el universitario español elige Internet y las redes sociales también en su tiempo de ocio. Tuenti y Facebook son las líderes, como ha mostrado el estudio sobre redes sociales, elaborado por Top Position (2011: web). Monge y Olabarri (2011: 80) aluden a otro estudio del observatorio The Cocktail Analysis, para explicar que "el porcentaje de la población internauta española usuaria de éstas se incrementó del 12\% al $33 \%$ en el caso de Tuenti y del $13 \%$ al $64 \%$ en el caso de Facebook". Según el mismo estudio, el $85 \%$ de los jóvenes españoles, de 16 a 25 años de edad, usa Tuenti frente a Facebook. Son los primeros adoptantes de las redes sociales y el grupo de población "que está construyendo sus dinámicas de relación en convivencia con ellas". También son los que están en la universidad y los profesores que les forman deben conocer lo que les gusta.

Internet también ayuda a los universitarios a trabajar en equipo y a encontrar trabajo. Mientras estudian o justo al abandonar las aulas. Las redes sociales profesionales, como LinkedIn, Xing o Job\&Talent, multiplican diariamente su número de usuarios, en España y en todo el mundo. Los estudiantes se ven obligados a utilizar la tecnología para no quedarse atrás. Es una revolución de lo profesional y lo educativo; en definitiva, de la inteligencia humana. Ésta ya no es "una especie de recurso mental de uso general, tamaño fijo y amorfo, que Dios o nuestros genes nos proporcionaron cuando nacimos, cuyo principal efecto era el de poner un techo de limitaciones a cuanto pudiéramos conseguir en la vida" (Claxton, 2005: 261). Hoy, la inteligencia es cambiante, aumentable, y dúctil, como comentábamos en las líneas anteriores. "Todos podemos ser mucho más inteligentes si desarrollamos nuestra capacidad para aprender" (Claxton, 2005: 261).

Aplicando esta información al alumnado y sobre todo al profesorado, y relacionándolo con los resultados sobre el estudio aquí presentado, comprobamos que también se puede aprender a través de Internet, del correo electrónico y de las redes sociales; a las que damos poco valor educativo inmerecidamente. Por ello, insistimos en la idea de que la enseñanza y la docencia universitaria del EEES deben aprender a aprovecharse de las nuevas tecnologías y de las funciones y potencialidades de las redes sociales. Porque las redes sociales están aquí para quedarse. No son una moda pasajera y como parte importante de la vida cotidiana de los jóvenes españoles, la universidad del EEES también debe acercarse a ellas.

Como prometíamos en la introducción, hemos relacionado los datos de un estudio real y riguroso sobre redes sociales, con la realidad de la docencia universitaria actual. Y a partir de ahí, planteamos utilizar los resultados del estudio para proponer algunos cambios o innovaciones educativas, que puedan ayudar a los docentes universitarios a acercarse mejor al alumnado: abrir un perfil en Facebook y/o Tuenti para cada asignatura, mantener ese perfil vivo con actualizaciones diarias y enlaces a webs interesantes, promover que los estudiantes puedan ser los community managers de ese perfil -por grupos y por un tiempo determinado-, incentivar que el alumnado también participe en el mantenimiento del perfil añadiendo enlaces y contenidos, convertir estas acciones en parte de la asignatura y no en un mero juego, y fomentar acciones para- 
lelas como la creación de blogs personales y temáticos, donde cada estudiante pueda centrarse en el asunto que más le guste o al que quiera dirigir su futuro profesional.

\section{Conclusiones}

Facebook y Tuenti ya no son solamente esas interfaces divertidas, de colores azul y verde, con fotografías donde encontramos a los amigos perdidos, o hablamos con los amigos actuales lo que no hemos podido, o querido, hablar físicamente y de tú a tú. En las redes sociales nos unimos a lo que nos gusta y nos llena. Seguimos a los famosos o a los artistas que admiramos; o nos conectamos a los perfiles de las empresas, productos o grupos que elegimos libremente, para sentirnos parte activa y necesaria de su devenir.

Las redes sociales no son una moda pasajera o una burbuja que pueda estallar y desaparecer mañana. Son la prueba del cambio radical de los paradigmas de identidad, de relación social, de comunicación, de enseñanza y de ocio, que citábamos desde el principio. Lo más individual y lo más social se han acoplado para demostrar a empresas e instituciones que ya no son tan poderosas y que necesitan de los usuarios de las redes sociales. Del mismo modo, las redes sociales ofrecen herramientas potenciales para promover la innovación educativa universitaria. Ha quedado demostrado que pueden ayudar a los docentes universitarios a acercarse mejor al alumnado. Como parte importante de la vida cotidiana de los jóvenes españoles, según los resultados de la empresa consultora Top Position (2011: web), la universidad del EEES también debe acercarse a ellas.

\section{Referencias bibliográficas}

ALADRO, Eva (2009): "Espacio Interpersonal, Sistemas de Conducta y Escaladas". CIC Cuadernos de Información y Comunicación, $\mathrm{n}^{\circ} 14$. Madrid, Servicio de Publicaciones de la Editorial Complutense, pp. 107-119.

ARRAS, Ana María de Guadalupe, TORRES, Carlos Arturo y GARCÍA-VALCÁRCEL, Ana (2011): "Los casos de la Universidad de Salamanca, España; Universidad Veracruzana y Universidad Autónoma de Chihuahua en México". Revista Latina de Comunicación Social, $\mathrm{n}^{\mathrm{o}}$ 66. Tenerife, Universidad de La Laguna, pp. 130-152.

CÁCERES, María Dolores, RUIZ, José Antonio y BRÄNDLE, Gaspar (2009): “Comunicación interpersonal y vida cotidiana. La presentación de la identidad de los jóvenes en Internet". CIC Cuadernos de Información y Comunicación, $\mathrm{n}^{\circ} 14$. Madrid, Servicio de Publicaciones de la Editorial Complutense, pp. 213-231.

CASTELLÓ, Araceli (2010): “¿Cuánto vale un fan? El reto de la medición de la audiencia en los social media". Pensar la Publicidad. Revista Internacional de Investigaciones Publicitarias, vol. $4, \mathrm{n}^{\mathrm{o}} 2$. Madrid, Servicio de Publicaciones de la Editorial Complutense, pp. 89-110.

CLAXTON, Guy (2005): “Aprendiendo a aprender: objetivo clave en el currículum del siglo XXI”. Traducción de Eva Aladro. CIC Cuadernos de Información y Co- 
municación, $\mathrm{n}^{\circ}$ 10. Madrid, Servicio de Publicaciones de la Editorial Complutense, pp. 259-265.

GALÁN, Elena y DEL PINO, Cristina (2010): “Jóvenes, ficción televisiva y nuevas tecnologías". Área Abierta, no 25. Madrid, Servicio de Publicaciones de la Editorial Complutense, pp. 1-17.

GÓMEZ NIETO, Begoña, TAPIA FRADE, Alejandro y DÍAZ CHICA, ÓSCAR (2012): "La comunicación corporativa a través de las páginas Web: el caso de las ONGs españolas". Revista de Comunicación Vivat Academia, $\mathrm{n}^{\mathrm{0}}$ 120, septiembre de 2012. Madrid, Universidad Complutense de Madrid, pp. 1-20.

LARRAÑAGA, Julio (2008): “Análisis económico de la evolución de las audiencias de los medios tradicionales e impacto de Internet en su consumo". Área Abierta, $\mathrm{n}^{\circ}$ 21. Madrid, Servicio de Publicaciones de la Editorial Complutense, pp. 1-18.

MATELLANES-LAZO, Mónica (2012): "Comparativa de contenidos enoturísticos en la web 2.0". Revista de Comunicación Vivat Academia, $\mathrm{n}^{\mathrm{o}}$ 120, septiembre de 2012. Madrid, Universidad Complutense de Madrid, pp. 21-43.

MONGE, Sergio y OLABARRI, María Elena: "Los alumnos de la UPV/EHU frente a Tuenti y Facebook: usos y percepciones". Revista Latina de Comunicación Social, $\mathrm{n}^{\circ}$ 66. Tenerife, Universidad de La Laguna, pp. 79-100.

PADILLA, Graciela, SEMOVA, Dimitrina y REQUEIJO, Paula: "Las motivaciones, estructura y usos de los correos electrónicos mediante internet: un estudio multidisciplinar". CIC Cuadernos de Información y Comunicación, $\mathrm{n}^{\circ}$ 16. Madrid, Servicio de Publicaciones de la Editorial Complutense, pp. 181-195.

TOP POSITION (2011): "Estudio jóvenes universitarios y redes sociales" en: http://posicionamientoenbuscadoreswebseo.es/estudio-jovenes-universitarios-yredes-sociales/

TORRES-SALINAS, Daniel (2008): "Mark Zuckerberg, fundador de Facebook, en la Universidad de Navarra". El profesional de la información, vol. 17, $\mathrm{n}^{\circ}$ 6. Barcelona, pp. 681-684.

\section{Graciela PADILla CASTILLO}

gracielapadilla@ccinf.ucm.es

Facultad de Ciencias de la Información (Universidad Complutense de Madrid). Despacho 543. Av. Complutense s/n. 28040 Madrid.

Profesora titular interina. Departamento de Periodismo III. Facultad de Ciencias de la Información (Universidad Complutense de Madrid). 